\title{
Avaliação da qualidade de amostras comerciais de folhas e tinturas de guaco
}

\author{
Flavia C. R. Alvarenga, ${ }^{1,2}$ Eliana de F. Garcia, ${ }^{2}$ Esther M. A. F. Bastos, ${ }^{1}$ Telma S. M. Grandi, ${ }^{2}$ \\ Maria Gorette R. Duarte*,1 \\ ${ }^{1}$ Divisão de Ciências Farmacêuticas, Fundação Ezequiel Dias (FUNED), Rua Conde Pereira Carneiro, 80, \\ Gameleira, 30510-010 Belo Horizonte-MG, Brasil, \\ ${ }^{2}$ Centro Universitário Newton Paiva, Av. Silva Lobo, 1720, Nova Granada, 30460-00 Belo Horizonte-MG, Brasil
}

\begin{abstract}
RESUMO: O mercado brasileiro de fitoterápicos encontra-se em crescente expansão, porém a falta de padronização e a má qualidade da matéria-prima vegetal constituem um problema crucial na produção de fitoterápicos de qualidade. Neste trabalho, foram avaliadas drogas vegetais constituídas de folhas (amostras A, B, C) e tinturas (amostras D, E, F) de guaco, adquiridas no Mercado Central e em farmácias de manipulação de Belo Horizonte, respectivamente. Realizaram-se testes físicoquímicos de caracterização organoléptica, determinação de matéria estranha, teores de água e cinzas totais para as amostras de drogas vegetais e caracterização organoléptica, medidas de pH e de densidade para as amostras de tinturas. Foram obtidos os perfis cromatográficos por CCD e CLAE-FR e fez-se a quantificação da cumarina (marcador químico), por CLAE-FR. As amostras analisadas apresentaram um ou mais dos parâmetros avaliados fora dos valores preconizados pela farmacopéia brasileira, evidenciando-se a necessidade da realização do controle de qualidade de matérias-primas vegetais, para se obter medicamentos fitoterápicos de qualidade, eficientes e seguros.
\end{abstract}

Unitermos: Guaco, controle de qualidade, cumarina, quantificação, CLAE-FR.

\begin{abstract}
Evaluation of the quality of commercial samples of leaves and tinctures of guaco". The Brazilian market of herbal medicine is in increasing expansion; however, the lack of standardization and the bad quality of the plant raw materials constitute a crucial problem for the production of quality herbal medicines. In this work, herbal drugs consisting of leaves (samples A, B, C) and tinctures (samples D, E, F) of guaco purchased in the Central Market and from pharmacies in Belo Horizonte, respectively, were evaluated. Physical-chemical tests such as organoleptic characterization, determination of foreign matter, total ash and water content were performed on the samples of herbal drugs. The organoleptic characterization, measures of $\mathrm{pH}$ and density of the tincture samples were also determined. TLC and RP-HPLC profiles of the herbal drugs and tinctures were obtained and the quantification of coumarin (used as a marker compound) by RPHPLC was determined. The analyzed samples showed one or more of the evaluated parameters outside of the values recognized by the Brazilian Pharmacopoeia. This demonstrates the need for the quality control of the plant raw materials, in order to guarantee quality and safety in herbal medicine.
\end{abstract}

Keywords: Guaco, quality control, coumarin, quantification, RP-HPLC.

\section{INTRODUÇÃO}

No Brasil, o mercado de fitoterápicos correspondia, em 1994, a um valor estimado em US\$ 355 milhões, o que representava 5,5\% do valor total das vendas de medicamentos, não se considerando a associação de princípios ativos de origem vegetal com aqueles de outra natureza. Em 1998, este mesmo mercado foi estimado em US\$ 566 milhões. É importante ressaltar a dificuldade na obtenção de informações sobre o volume real movimentado no país, devido ao mercado interno informal forte, que não é contabilizado nem controlado, onde plantas, sementes, raízes e folhas são vendidas nas ruas e feiras de muitas cidades (Alves,
2004). O crescimento do mercado de fitoterápicos, no Brasil, é da ordem de $15 \%$ ao ano, enquanto o mercado de medicamentos sintéticos cresce em torno de 3 a $4 \%$ (Febrafarma, 2007).

O aumento no uso de fitoterápicos pela população mundial também tem se traduzido em preocupação com a qualidade de tais produtos, devido aos problemas comumente encontrados referentes à autenticidade, pureza e composição química das matérias-primas vegetais que contribuem para um fitoterápico de má qualidade (Carvalho et al., 2008; Silveira et al., 2008). No mercado brasileiro, esses problemas são freqüentemente encontrados em várias regiões do país (Borella \& Fontoura, 2002; Amaral 
et al., 2003a; Melo et al., 2004; Marliére et al., 2008; Veiga-Junior, 2008).

No Brasil, várias espécies nativas têm sido largamente empregadas pela população com fins medicinais, entre estas podem ser citadas espécies de Baccharis (carqueja), Bauhinia (pata-de-vaca), Cecropia (embaúba), Maytenus (espinheira-santa), Passiflora (maracujazeiro) e Mikania (guaco) (Reis et al., 2004; Agra et al., 2007; 2008).

O gênero Mikania Willdenow foi estabelecido em 1803 e pertence à família Asteraceae, uma das maiores famílias do reino vegetal. O gênero compreende cerca de 600 espécies, distribuídas em regiões tropicais da América, África e Ásia, das quais 152 ocorrem no Brasil (Oliveira et al., 1994).

Dentre as espécies de Mikania Willdenow, pertencentes à secção Globosae Robinson destacam-se algumas de utilização medicinal, a saber, Mikania cordifolia Willd., Mikania scandens Willd., Mikania officcinalis Mart., Mikania opifera D. C., Mikania glomerata Sprengel e Mikania laevigata Sch. Bip. ex Baker (Neves \& Sá, 1991; Oliveira et al., 1994). As espécies mais utilizadas são $M$. glomerata e $M$. laevigata, conhecidas popularmente como guaco. $M$. glomerata consta da $1^{\mathrm{a}}$ edição da Farmacopéia Brasileira e da lista de registro simplificado de fitoterápicos (RE $\mathrm{n}^{\circ}$ 89) (Pharmacopéia dos Estados Unidos do Brasil, 1926; Brasil, 2004; Brandão et al., 2008) e $M$. laevigata encontra-se descrita no sexto fascículo da farmacopéia brasileira $4^{\mathrm{a}}$ edição (Farmacopéia Brasileira, 2005). A cumarina (1,2-benzopirona) é o marcador químico das duas espécies (Brasil, 2004; Farmacopéia Brasileira, 2005), sendo responsável, em parte, pelo efeito terapêutico observado (Oliveira et al., 1986/1987; Leite, 1993; Silva et al., 2008).

As espécies $M$. glomerata e $M$. laevigata, amplamente empregadas pela população, apresentam semelhanças morfoanatômicas, sendo comercializadas indistintamente. As folhas são empregadas na medicina popular para tratar doenças do trato respiratório, como asma e bronquite, além de serem adjuvantes no combate à tosse (Lucas, 1941; Gupta, 1995; Cabral et al., 2001; Amaral et al., 2003b).

Este trabalho foi desenvolvido com o objetivo de avaliar a qualidade das folhas e tinturas de guaco, comercializadas em Belo Horizonte, verificando se as amostras encontravam-se de acordo com as exigências da legislação específica.

\section{MATERIAL E MÉTODOS}

\section{Amostras avaliadas}

Três amostras de folhas secas de guaco (amostras A, B, C) e três amostras de tinturas de guaco (amostras D, E, F) foram adquiridas no Mercado Central e em farmácias de manipulação de Belo Horizonte, respectivamente.

\section{Droga vegetal padrão}

Empregou-se como droga vegetal padrão (Dp) a espécie $M$. glomerata coletada no Horto de Plantas Medicinais do Centro Universitário Newton Paiva e identificada pela botânica Telma Sueli Mesquita Grandi.

\section{Preparo da tintura padrão}

A tintura padrão (Tp) foi preparada a 20\% (p/v) pelo processo de percolação, conforme descrito na $1^{\text {a }}$ edição da Farmacopéia Brasileira (Pharmacopéia dos Estados Unidos do Brasil, 1926).

\section{Amostragem}

A amostragem foi efetuada por quarteamento como descrito na Farmacopéia Brasileira para lotes menores de $10 \mathrm{Kg}$ de folhas, obtendo-se, como amostra final, $250 \mathrm{~g}$ de amostra (Farmacopéia Brasileira, 2000).

\section{Caracterização organoléptica}

As amostras de drogas vegetais e tinturas foram analisadas quanto à cor, odor e aspecto, a partir dos parâmetros descritos por Lucas (1941) e pela Brasileira (Pharmacopéia dos Estados Unidos do Brasil, 1926; Farmacopéia Brasileira, 1988). Para melhor visualização das características, as drogas vegetais e tinturas foram transferidas para folhas de papel branco e tubos de ensaio, respectivamente, e observadas sob luz natural. Para determinação da coloração das tinturas, utilizou-se como referência os padrões de cores descritos por Melo (1946).

\section{Determinação de material estranho}

As amostras ( $250 \mathrm{~g})$ foram submetidas a uma tamisação seqüencial (MESH 10, 20, 32, 80 e fundo). As frações retidas em cada tamis foram pesadas e armazenadas separadamente. Os materiais estranhos foram retirados manualmente, a olho nu, sendo posteriormente transferidos para placas de Petri e observados ao microscópio esterioscópio. Os diferentes tipos de materiais estranhos foram separados, pesados e os percentuais calculados (Farmacopéia Brasileira, 2000).

\section{Determinação de água}

Para a determinação do teor de água utilizouse o método gravimétrico descrito na Farmacopéia Brasileira (Farmacopéia Brasileira, 2000). As análises foram realizadas em triplicata para todas as amostras e os resultados expressos em porcentagem, em relação à 
droga vegetal.

\section{Determinação de cinzas totais}

Na determinação do teor de cinzas totais utilizou-se o método descrito na Farmacopéia Brasileira (Farmacopéia Brasileira, 2000). As análises foram realizadas em triplicata e o teor foi calculado em relação à droga vegetal, sendo o resultado expresso em porcentagem.

\section{Determinação do pH}

Para a avaliação do $\mathrm{pH}$, realizada em triplicata, utilizou-se aparelho Digimed-mod DM 20, aferido com soluções tampão de referência, $\mathrm{pH} 4,0$ e 7,0 (Farmacopéia Brasileira, 1988).

\section{Determinação da densidade}

Empregou-se o método do picnômetro (Farmacopéia Brasileira, 1988).

\section{Obtenção dos perfis cromatográficos por CCD}

Para as amostras de drogas vegetais, fezse extração prévia em refluxo conforme monografia do guaco-cheiroso (Farmacopéia Brasileira, 2005), enquanto as tinturas foram aplicadas diretamente sobre a cromatoplaca. Para a obtenção dos perfis cromatográficos das drogas vegetais e tinturas utilizaram-se cromatoplacas de alumínio recobertas por sílica gel 60G $\mathrm{F}_{254}$ Merck, sendo empregado como eluente tolueno-diclorometano-acetona (45:25:30) e solução etanólica de $\mathrm{KOH}$ a $5 \%$, sob luz $\mathrm{UV}_{365 \mathrm{~nm}}$, como revelador. A cumarina (1,2-benzopirona, Sigma) foi usada como padrão. Os fatores de retenção $\left(R_{f}\right)$ foram calculados para as manchas fluorescentes observadas.

\section{Quantificação da cumarina por CLAE-FR}

As análises foram realizadas em cromatógrafo líquido Merck-Hitachi composto de bomba L-6200A, injetor automático AS-2000A, detector UV-VIS L-4250 e integrador D-2500. Empregou-se coluna LiChrocartLiChrospher $100 \mathrm{RP}-18(250 \mathrm{x} 4 \mathrm{~mm}, 5 \mu \mathrm{m})$ a $40{ }^{\circ} \mathrm{C}$, fluxo de $0,5 \mathrm{~mL} / \mathrm{min}$., detecção $\mathrm{UV}_{275 \mathrm{~nm}}$, eluição isocrática $\left(\mathrm{H}_{2} \mathrm{O}-\mathrm{MeOH}\right.$ 53:47) (Aboy et al., 2000). As amostras de drogas vegetais foram extraídas, sob refluxo, com $\mathrm{H}_{2} \mathrm{O}-\mathrm{EtOH}$ (1:1) de acordo com metodologia descrita por Aboy et al. (2000) e para as amostras de tinturas fizeram-se apenas diluições adequadas. Posteriormente, as amostras foram centrifugadas (10.000 r.p.m., $10 \mathrm{~min}$.) e o sobrenadante foi empregado nas análises $(20 \mu \mathrm{L})$. As amostras foram preparadas em triplicata, fazendose três injeções para cada triplicata. Na quantificação utilizou-se a cumarina (1,2-benzopirona, Sigma) como padrão externo. Para a construção da curva de calibração preparou-se uma solução estoque a 0,5 mg/ $\mathrm{mL}$ em $\mathrm{MeOH}$ e alíquotas desta foram diluídas em $\mathrm{H}_{2} \mathrm{O}-\mathrm{MeOH}$ (53:47), obtendo-se soluções a 0,$005 ; 0,01$; 0,$02 ; 0,04$ e $0,08 \mathrm{mg} / \mathrm{mL}$ (Celeghini et al., 2001), que foram injetadas em quintuplicata. Calculou-se o teor da cumarina a partir da equação da reta e o resultado foi expresso pela média das determinações em gramas de cumarina por $100 \mathrm{~g}$ da amostra, considerando-se a determinação de água (\%).

\section{Obtenção dos perfis cromatográficos por CLAE-FR}

As análises foram realizadas no equipamento descrito anteriormente, empregando-se coluna LiChrocart-LiChrospher $100 \mathrm{RP}-18$ (250 x $4 \mathrm{~mm}, 5$ $\mu \mathrm{m}$ ) a $40{ }^{\circ} \mathrm{C}$, fluxo de $1,0 \mathrm{~mL} / \mathrm{min}$., detecção $\mathrm{UV}_{210 \mathrm{~nm}}$, eluição isocrática $\left(\mathrm{CH}_{3} \mathrm{CN}-\mathrm{H}_{2} \mathrm{O}\right.$ 15:85). As amostras de drogas vegetais foram extraídas em ultra-som, conforme metodologia descrita por Celeghini et al (2001), e as amostras de tinturas foram apenas diluídas adequadamente. A seguir, as amostras foram centrifugadas (10.000 r.p.m., $10 \mathrm{~min}$.) e o sobrenadante foi empregado nas análises $(20 \mu \mathrm{L})$. Fez-se uma única injeção.

\section{Análise dos laudos das tinturas}

Os laudos dos fornecedores foram analisados quanto à presença das seguintes informações: nome do fabricante, nomes popular e científico da espécie, parte utilizada, número do lote, datas de fabricação e validade, relação droga:solvente extrator, ensaios físico-químicos e suas especificações e referências bibliográficas.

\section{RESULTADOS E DISCUSSÃO}

Na aquisição das amostras não se fez referência ao nome científico da espécie vegetal, sendo estas adquiridas apenas pelo nome popular “guaco”. Os resultados dos testes físico-químicos estão apresentados na Tabela 1. Todas as amostras de drogas vegetais apresentaram odor aromático característico de cumarina e coloração esverdeada, conforme descrito por Lucas (1941) e pela Farmacopéia Brasileira (Farmacopéia Brasileira, 2005). Observou-se grande diferença no estado de fragmentação das folhas. As folhas das amostras A e C apresentaram-se inteiras e da amostra B, fragmentadas, detectando-se a presença de pó (Figura 01). As tinturas analisadas exibiram odor aromático próprio, mas apresentaram cores e aspectos diferentes. A tintura D apresentou aspecto turvo e coloração marrom-avermelhada, a tintura E mostrou-se límpida e marrom-esverdeada, a tintura F, límpida e marrom a marrom-escuro. A tintura padrão (Tp) apresentou aspecto límpido e coloração marrom-esverdeada. Pôdese observar, assim, que a tintura E apresentou coloração 
Tabela 1. Testes físico-químicos realizados para as amostras de drogas vegetais e tinturas.

\begin{tabular}{|c|c|c|c|c|c|c|}
\hline Amostra $^{1}$ & Caracteres organolépticos & $\begin{array}{c}\text { Material estranho }{ }^{2} \\
(\%)\end{array}$ & $\begin{array}{c}\text { Cinzas totais }^{3} \\
(\%)\end{array}$ & $\begin{array}{c}\text { Teor de água }{ }^{4} \\
\text { (\%) }\end{array}$ & $\mathrm{pH}$ & $\begin{array}{l}\text { Densidade } \\
\mathrm{g} / \mathrm{mL}\end{array}$ \\
\hline A & $\begin{array}{l}\text { Folhas secas inteiras de cor } \\
\text { verde, com presença de caules, } \\
\text { odor característico }\end{array}$ & 5,44 & $16,98 \pm 0,21$ & $9,55 \pm 0,10$ & - & - \\
\hline B & $\begin{array}{l}\text { Folhas secas inteiras de cor } \\
\text { verde, fragmentadas com } \\
\text { presença de pó e caules, odor } \\
\text { característico }\end{array}$ & 17,82 & $12,32 \pm 0,27$ & $11,11 \pm 0,04$ & - & - \\
\hline $\mathrm{C}$ & $\begin{array}{l}\text { Folhas secas inteiras de cor } \\
\text { verde, com presença de grande } \\
\text { quantidade de caules, odor } \\
\text { característico }\end{array}$ & 45,29 & $13,58 \pm 0,16$ & $12,71 \pm 0,15$ & - & - \\
\hline $\mathrm{D}$ & $\begin{array}{l}\text { Cor marrom avermelhada, } \\
\text { turva/opaca, odor } \\
\text { característico }\end{array}$ & - & & & 5,77 & 0,965 \\
\hline $\mathrm{E}$ & $\begin{array}{l}\text { Cor marrom esverdeada, } \\
\text { translúcida, odor característico }\end{array}$ & - & & & 6,34 & 0,895 \\
\hline $\mathrm{F}$ & $\begin{array}{l}\text { Cor marrom a marrom escura, } \\
\text { translúcida, odor característico }\end{array}$ & - & & & 6,10 & 0,909 \\
\hline
\end{tabular}

(1) A, B, C: drogas vegetais; C, D e E: tinturas; (2) máx. 2\% (Farmacopéia Brasileira, 2005); (3) máx. 15\% (Farmacopéia Brasileira, 2005); (4) máx. 10\% (Farmacopéia Brasileira, 2005); (-) teste não realizado

mais próxima à $\mathrm{Tp}$, no entanto, os resultados obtidos para as tinturas $\mathrm{D}$ e $\mathrm{F}$ não foram condizentes com aqueles encontrados para $\mathrm{Tp}$ e descritos na literatura (Pharmacopéia dos Estados Unidos do Brasil, 1926; Lucas, 1941) (Figura 1). A coloração das tinturas foi avaliada de acordo com o padrão descrito por Melo (1946).

Considerando-se os limites preconizados na monografia do guaco-cheiroso (Farmacopéia Brasileira, 2005) para os ensaios de material estranho (máx. 2\%), cinzas totais (máx. 15\%) e umidade (máx. 10\%), constatou-se que as amostras avaliadas apresentaram valores superiores aos estabelecidos, excetuando-se o teor de água da amostra A $(9,55 \%)$ e os teores de cinzas totais das amostras B e C (12,32 e $13,58 \%$, respectivamente). Ressalta-se o alto teor de material estranho encontrado na amostra C $(45,29 \%)$, constituído por fragmentos de insetos e principalmente caules (Figura 2). Os valores de $\mathrm{pH}$ observados para as amostras de tinturas variaram de 5,77 a 6,34; mostrando-

Tabela 2. Resultados da quantificação da cumarina nas amostras de drogas vegetais e tinturas.

\begin{tabular}{cc}
\hline Amostras & Cumarina $^{1}(\%)$ \\
\hline Dp & $0,71 \pm 0,23$ \\
A & $0,52 \pm 0,06$ \\
B & $0,15 \pm 0,01$ \\
C & ND \\
Tp & $0,176 \pm 0,002$ \\
D & $0,024 \pm 0,0003$ \\
E & $0,015 \pm 0,01$ \\
F & $0,072 \pm 0,001$
\end{tabular}

(1) resultado expresso pela média, sendo $\mathrm{n}=9$; (ND) não detectado se de acordo com aqueles encontrados por Osorio (2002) para tinturas de guaco comercializadas em São Paulo $(5,46$ a 6,33$)$. Na determinação da densidade os valores obtidos variaram de 0,895 a 0,965 g/mL. Estes resultados encontram-se dentro da faixa de densidade de 0,88 a 0,96 g/mL, descrita por Osório (2002)

Nos perfis cromatográficos por $\mathrm{CCD}$, as amostras de drogas vegetais $A, B$ e padrão (Dp) e de tinturas D, E, F e Tp apresentaram mancha fluorescente verde referente à cumarina $(\mathrm{Rf}=0,81)$, marcador químico descrito para as espécies $M$. glomerata e M. laevigata (Brasil, 2004; Farmacopéia Brasileira, 2005). Porém, na droga vegetal $C$ a cumarina não foi detectada (FIGURA 3). Detectou-se ainda, em todas as amostras, uma mancha fluorescente verde de $\mathrm{Rf}=0,34$, provavelmente referente ao ácido o-cumárico, conforme monografia do guaco-cheiroso (Farmacopéia Brasileira, 2005).

Na quantificação da cumarina por CLAE-FR, a equação da reta obtida por regressão linear para a curva padrão foi $y=2.10^{7} \mathrm{x}+769,09$, onde $\mathrm{y}$ representa a área dos picos (mAU) e $\mathbf{x}$ a concentração de cumarina $(\mathrm{mg} / \mathrm{mL})$. O coeficiente de correlação (r) obtido foi de 0,9999, denotando comportamento linear na faixa de concentração avaliada. As amostras A (0,52\%), B $(0,15 \%)$ e $\mathrm{Dp}(0,71 \%)$ (Tabela 2$)$ apresentaram teores de cumarina acima do mínimo de $0,1 \%$ preconizado pela farmacopéia brasileira (Farmacopéia Brasileira, 2005). As espécies M. glomerata e M. laevigata são empregadas popularmente, de forma indistinta, para os mesmos fins medicinais. Assim, utilizou-se como parâmetro o teor mínimo de cumarina exigido pela monografia do guaco-cheiroso ( $M$. laevigata), visto que não existe limite farmacopeico para este marcador nas 

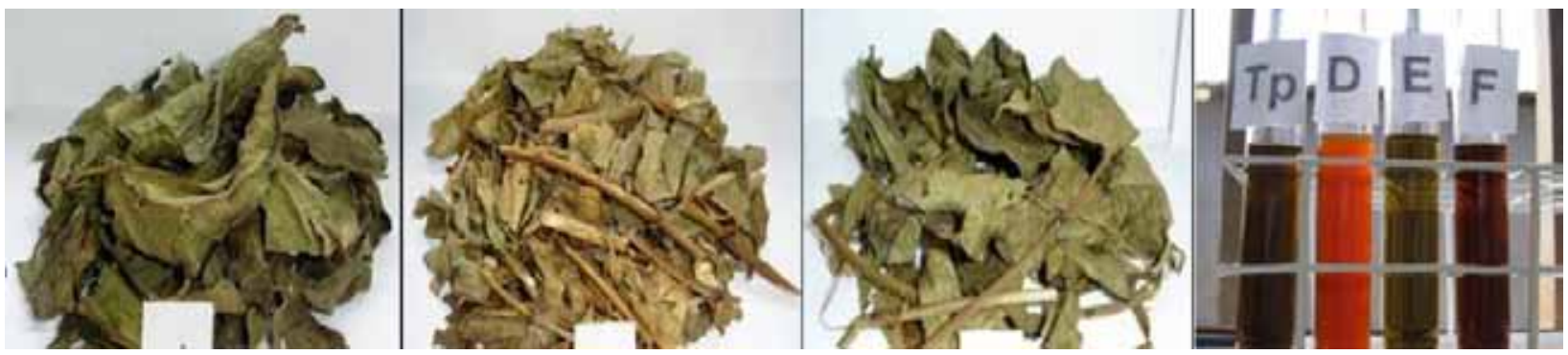

Figura 1. Amostras de folhas de guaco (A, B e C) e tinturas (Tp, D, E e F) avaliadas.
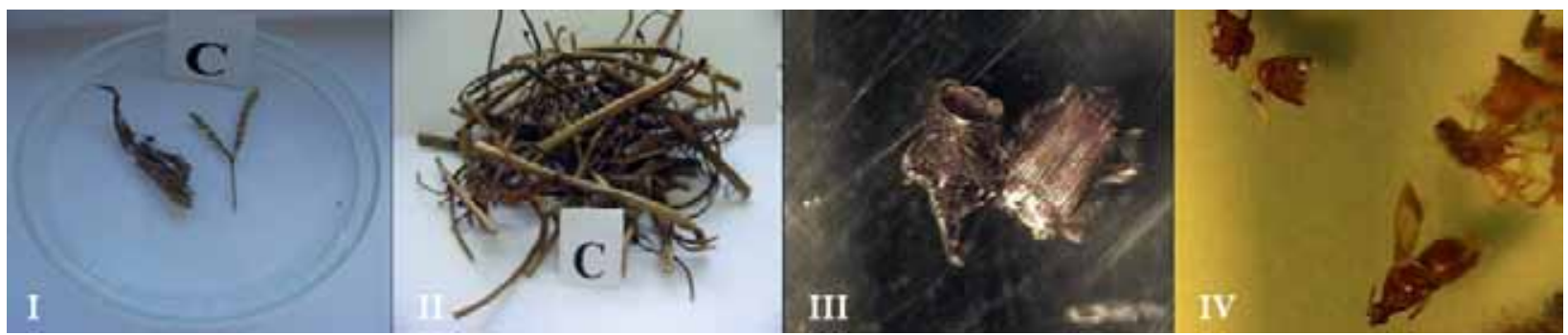

Figura 2. Material estranho encontrado na amostra C.

I-Folhas de outros vegetais; II-Caules; III-Besouro; IV-Fragmentos de insetos.

folhas de $M$. glomerata. No cromatograma da amostra C não foi detectado nenhum pico com TR próximo de 6 min., relativo à cumarina, confirmando o resultado obtido na CDD (Figura 3).

Os teores de cumarina nas tinturas $\mathrm{D}, \mathrm{E}, \mathrm{F}$ e Tp foram de $0,024 \% ; 0,015 \% ; 0,072 \%$ e $0,176 \%$, respectivamente (Tabela 2). Observou-se uma variação nos teores de cumarina encontrados decorrente da falta de padronização da relação droga:solvente extrator empregada no preparo das tinturas. Esperava-se um menor teor de cumarina para a tintura D que exibia uma menor proporção droga:solvente extrator $(4 \% \mathrm{p} / \mathrm{v})$, no entanto, este foi superior ao da tintura E, preparada a $20 \%$. Por sua vez, a tintura $\mathrm{E}$ apresentou teor de cumarina muito aquém daquele apresentado pela Tp, preparada na mesma concentração. Para a tintura F, preparada a $25 \%$, o teor encontrado foi inferior ao de Tp, cuja relação droga: solvente extrator era de $20 \%$. Apesar de não existir um valor mínimo preconizado para o teor de cumarina em tinturas de guaco, a quantificação do marcador químico torna-se importante, já que podese inferir sobre a qualidade da matéria-prima vegetal utilizada na preparação, bem como a eficiência do método de extração empregado.

Os perfis cromatográficos por CLAE-FR (Figuras 4 e 5) foram determinados com a finalidade de se conhecer a composição qualitativa e comparar os perfis das drogas vegetais e tinturas. As amostras
Verde fluorescente

$$
\mathrm{R}_{\mathrm{f}}=0,81
$$

Azul fluorescente

$$
\mathrm{R}_{\mathrm{f}}=0,64
$$

Verde fluorescente $\mathrm{R}_{\mathrm{f}}=0,34$
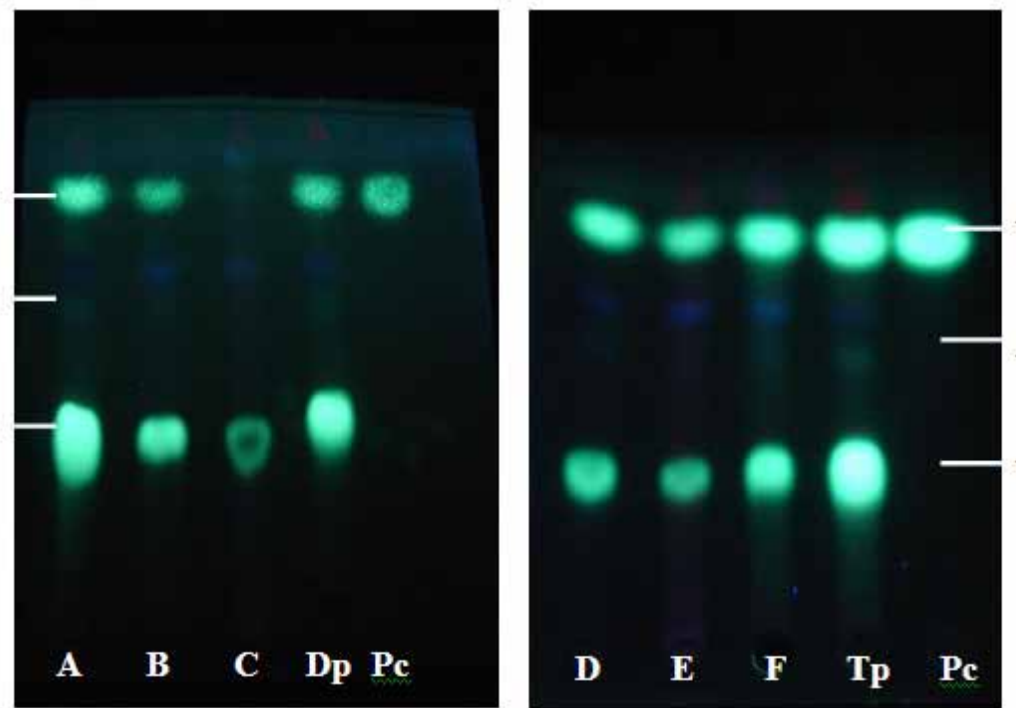

Verde fluorescente

$$
R_{f}=0,81
$$

Azul fluorescente

$R_{f}=0,57$

Verde fluorescente

$\mathrm{R}_{f}=0.34$ 


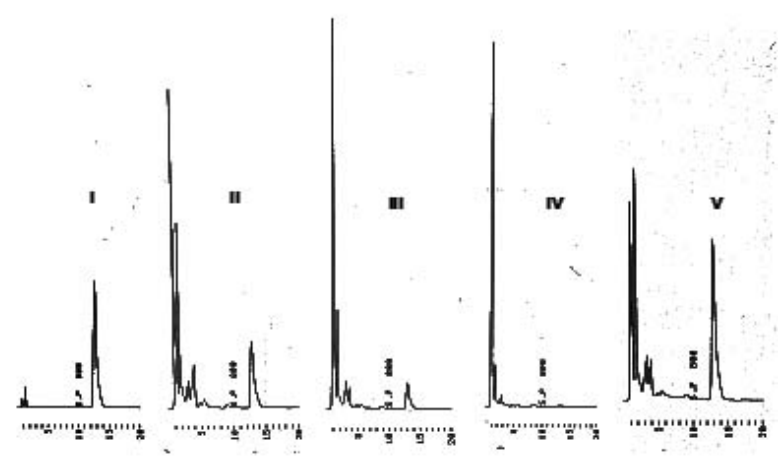

Figura 4. Perfis cromatográficos, obtidos por CLAE-FR, para as amostras de drogas vegetais.

I - Cumarina $(0,04 \mathrm{mg} / \mathrm{mL})$; II - Droga vegetal A; III - Droga vegetal B; IV - Droga vegetal C; V - Droga vegetal padrão. Condições cromatográficas: vide Material e Métodos.

avaliadas apresentaram perfis semelhantes, observandose predominância de compostos polares, com TR menores que 5 min., o que pode ser explicado pelo uso de solvente extrator hidroalcoólico. Dentre as amostras de drogas vegetais analisadas apenas a droga vegetal C não apresentou pico com TR próximo de 12 min., referente à cumarina, corroborando o resultado obtido na quantificação.

A análise dos laudos das tinturas permitiu constatar para todas as amostras a presença de informações essenciais, tais como, nome do fabricante, lote, nomes popular e científico da droga vegetal, caracteres organolépticos (cor, odor e sabor), datas de validade e fabricação, forma farmacêutica, valor de $\mathrm{pH}$, densidade, relação droga:solvente extrator e solubilidade em alguns solventes. O laudo da tintura D apresentou ainda informações adicionais sobre controle microbiológico, resíduo seco, reações de identificação de polifenóis e flavonóides, teor alcoólico e a bibliografia consultada. O laudo da amostra E informou sobre o emprego da tintura, classe toxicológica, reações adversas, interações, resíduo seco, índice de refração, cinzas totais, cinzas insolúveis em $\mathrm{HCl}$ e análise cromatográfica em camada delgada. A avaliação dos laudos destacou a falta de padronização das informações presentes, a ausência de bibliografia oficial, a variação nos tipos de testes realizados e a diferença nos prazos de validade das tinturas (2 a 3 anos).

\section{CONCLUSÃO}

As amostras analisadas apresentaram um ou mais dos parâmetros avaliados fora dos valores preconizados pela farmacopéia brasileira, evidenciandose a baixa qualidade das drogas vegetais e tinturas comercializadas. Torna-se, então, necessária a realização do controle de qualidade das matérias-primas vegetais, visando a obtenção de medicamentos fitoterápicos padronizados, eficientes e seguros, segundo as normas estabelecidas pela legislação brasileira.

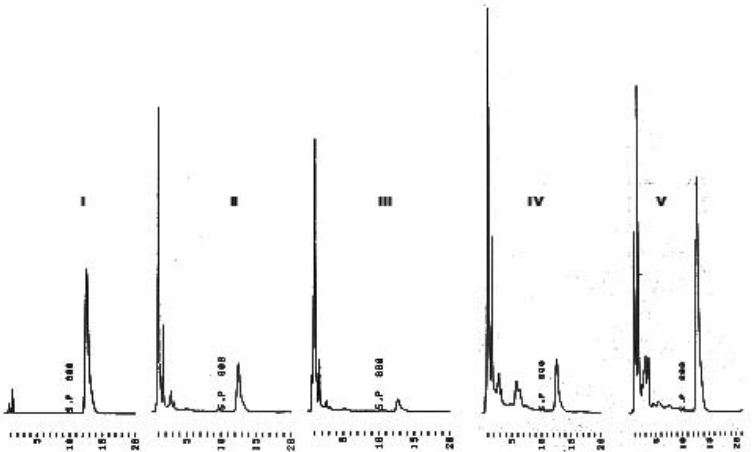

Figura 5. Perfis cromatográficos, obtidos por CLAE-FR, para as amostras de tinturas.

I - Cumarina $(0,04 \mathrm{mg} / \mathrm{mL})$; II - Tintura D; III - Tintura E; IV Tintura F; V - Tintura padrão. Condições cromatográficas: vide Material e Métodos.

Apesar da farmacopéia brasileira $1^{\mathrm{a}}$ edição preconizar a utilização de tintura de guaco a $20 \%$ p/v, observaram-se concentrações diferentes para as tinturas comercializadas nas farmácias de manipulação de Belo Horizonte, o que poderia acarretar variações nos efeitos farmacológicos esperados. A quantificação da cumarina nas amostras de tinturas analisadas mostrou uma incoerência entre a concentração da tintura e o teor de cumarina, já que a tintura com maior relação droga:solvente extrator, tintura $\mathrm{F}$ (25\%), não apresentou o maior teor de cumarina. A falta de padronização observada no conteúdo dos laudos das tinturas pode ser reflexo da ausência de uma legislação clara e específica, que regulamente as informações contidas em um laudo.

Nos perfis cromatográficos por CCD e CLAEFR, assim como na análise quantitativa, a cumarina não foi detectada na amostra de droga vegetal C, estando esta ausente ou em quantidade não detectável. Considerandose que a cumarina é o marcador químico do guaco, esta ausência pode sugerir que a amostra $C$ era constituída de matéria-prima de má qualidade ou mesmo que não se referia ao guaco. A utilização desta droga não levaria ao efeito terapêutico desejado, visto ser a cumarina a responsável, em parte, pela ação broncodilatadora.

\section{AGRADECIMENTOS}

À FAPEMIG pela concessão de bolsa BIC (F.C.R.A.), e aos professores Geraldo Célio Brandão (UFMG) e Fernão Castro Braga (UFMG) pela colaboração nas análises por CLAE.

\section{REFERÊNCIAS}

Aboy AL, Petrovik GOPR, Langeloh VLB 2000. Desenvolvimento de soluções extrativas de Mikania glomerata Sprengel (Asteraceae), guaco. Rev Bras Ciênc Farm 36: 165-172.

Agra MF, França PF, Barbosa-Filho JM 2007. Synopsis of the plants known as medicinal and poisonous in Northeast of Brazil. Rev Bras Farmacogn 17: 114-140. 
Agra MF, Silva KN, Basílio IJLD, França PF, Barbosa-Filho JM 2008. Survey of medicinal plants used in the region Northeast of Brazil. Rev Bras Farmacogn 18: 472508.

Alves FNR 2004. Desafios para o desenvolvimento de fitomedicamentos no Brasil no contexto da indústria farmacêutica. Rio de Janeiro, 249p. Dissertação de Mestrado - Escola Nacional de Saúde Pública Sergio Arouca, Fundação Oswaldo Cruz.

Amaral FMM, Coutinho DF, Ribeiro MNS, Oliveira MA 2003a. Avaliação da qualidade de drogas vegetais comercializadas em São Luís/Maranhão. Rev Bras Farmacogn 13: 27-30.

Amaral RR, Arcenio Neto F, Carvalho ES, Teixeira LA, De Araújo GL, Sharapin N, Testa B, Gnerre C, Rocha L 2003b. Avaliação da atividade IMAO e antibacteriana de extratos de Mikania glomerata Sprengel. Rev Bras Farmacogn 13: 24-27.

Borella JC, Fontoura A 2002. Avaliação do perfil cromatográfico e do teor de flavonóides em amostras de Baccharis trimera (Less.) DC. Asteraceae (carqueja) comercializadas em Ribeirão Preto, SP, Brasil. Rev Bras Farmacogn 12: 63-67.

Brandão MGL, Zanetti NNS, Oliveira GRR, Goulart LO, MonteMor RLM 2008. Other medicinal plants and botanical products from the first edition of the Brazilian Official Pharmacopoeia. Rev Bras Farmacogn 18: 127-134.

Brasil 2004. Ministério da Saúde. Agência Nacional de Vigilância Sanitária, Resolução - RE no 89, de 16 de março de 2004. Lista de Registro Simplificado de Fitoterápicos. Brasília.

Cabral LM, Santos TC, Alhaique F 2001. Development of a profitable procedure for the extration of 2-H-benzopyran-2-one (coumarin) from Mikania glomerata. Drug Dev Ind Pharm 27: 103-106.

Carvalho ACB, Balbino EE, Maciel A, Perfeito JPS 2008. Situação do registro de medicamentos fitoterápicos no Brasil. Rev Bras Farmacogn 18: 314-319.

Celeghini RMS, Vilegas JHY, Lanças FM 2001. Extration and quantitative HPLC analysis of coumarin in hidroalcholic extratcts of Mikania glomerata Spreng. (guaco) leaves. J Braz Chem Soc 12: 706-709.

Farmacopéia Brasileira 1988. 4. ed. São Paulo: Editora Atheneu, parte I.

Farmacopéia Brasileira 2000. 4. ed. São Paulo: Editora Atheneu, parte II, segundo fascículo.

Farmacopéia Brasileira 2005. 4. ed. São Paulo: Editora Atheneu, parte II, sexto fascículo.

Febrafarma 2007. http://www.febrafarma.org.br, acessada em julho de 2007.

Gupta MP 1995. 270 Plantas Medicinales Iberoamericanas. Santa Fé de Bogotá: Convênio Andrés Belho.

Leite MGR, Souza CL, Silva MAM 1993. Estudo farmacológico comparado de Mikania glomerata Sprengel (guaco), Justicia pectoris Jacq (anador) e Torresea cearensis Fr. All (cumaru). Rev Bras Farm 74: 12-15.

Lucas V 1941. Estudo farmacognóstico do guaco: Mikania glomerata Sprengel-Composta. Rev Flora Med 9: 101-132.

Marliére LDP, Ribeiro AQ, Brandão MGL, Klein CH, Acurcio FA 2008. Utilização de fitoterápicos por idosos: resultados de um inquérito domiciliar em Belo Horizonte (MG), Brasil. Rev Bras Farmacogn 18 (Supl.): 754-760.

Melo MS 1946 Caracteres organolépticos de alimentos e bebidas. Rev Inst Adolf Lutz 4: 77-91.

Melo JG, Nascimento VT, Amorim ELC, Andrade Lima CS, Albuquerque UP 2004. Avaliação da qualidade de amostras comerciais de boldo (Peumus boldus Molina), pata-de-vaca (Bauhinia spp.) e ginco (Ginkgo biloba L.). Rev Bras Farmacogn 14: 111-120.

Neves JL, Sá MFA 1991. Contribuição ao estudo de plantas medicinais Mikania glomerata Sprengel. Rev Bras Farm 72: 42-47.

Oliveira F, Akisue G, Akisue MK, Mancini B, Chumzum M 1986/1987. Morfodiagnose do axófito do guaco Mikania glomerata Sprengel. Rev Ciênc Farm 8/9: 11-24.

Oliveira F, Saito ML, Garcia LO 1994. Morfologia externa das partes áreas e anatomia foliar das espécies brasileiras de Mikania Willdenow seç̧ão Globosea Robinson visão farmacognóstica. Lecta 12: 23-65.

Osório AC 2002. Controle de qualidade do extrato fluido e tintura de guaco (Mikania glomerata Sprengel). São Paulo, 121 p. Tese de Doutorado - Faculdade de Ciências Farmacêuticas, Universidade de São Paulo.

Pharmacopéia dos Estados Unidos do Brasil 1926. 1.ed. São Paulo: Companhia Editora Nacional.

Reis S, Mariot A, Steenbock W 2004. Diversidade e domesticação de plantas medicinais. In: Simões CMO et al. Farmacognosia: da planta ao medicamento. 5. ed. Florianópolis/Porto Alegre: UFSC/UFRGS, p.4569.

Silva CR, Gomes VS, Kulkamp IC, Kanis LA 2008. Método espectroscópico para determinação de cumarina em xarope de Mikania glomerata Sprengel. Rev Bras Farmacogn 18: 594-599.

Silveira PF, Bandeira MAM, Arrais PSD 2008. Farmacovigilância e reações adversas às plantas medicinais e fitoterápicos: uma realidade. Rev Bras Farmacogn 18: 618-626.

Veiga-Junior VF 2008. Estudo do consumo de plantas medicinais na Região Centro-Norte do Estado do Rio de Janeiro: aceitação pelos profissionais de saúde e modo de uso pela população. Rev Bras Farmacogn 18: 308-313. 